\title{
Ionisation Structure of NGC 6543
}

E. Pérez ${ }^{1,2}$, L. Cuesta ${ }^{3}$, D. Axon ${ }^{1}$ and A. Robinson ${ }^{4}$

${ }^{1}$ Space Telescope Science Institute, Baltimore, ; ${ }^{2}$ Instituto de Astrofísica de Andalucía;

${ }^{3}$ Instituto de Astrofísica de Canarias, La Laguna; ${ }^{4}$ University of Hertfordshire, Hartfield

We have obtained high spectral resolution, high signal-to-noise ratio longslit spectra of a sample of Planetary Nebulae with the purpose of studying their physical conditions, chemical composition and ionisation structure. In particular, we are interested in disentangling the effects of radiation and shock ionisation.

Here we present a sample of the data obtained for NGC 6543 along position angles $5^{\circ}$ and $20^{\circ}$. We cover the full wavelength, range from the atmospheric cutoff at $3000 \AA$, up to $1.06 \mu \mathrm{m}$ with a dispersion of $0.85 \AA$ /pixel.

From the corresponding two-dimensional spectroscopic frames for the two position angles it is already obvious how some emission lines have a structure indicative of shock ionisation; the brightest of these include [OII]3727,7325, [SII]4068,6725, [NI]5198, [NII]$5755,6548,6583$, [OI]6300,6364, and other fainter ones. Some lines, such as [SIII]6312,9069,9532 , show a intensity structure which indicates that it is both shock and photo-ionised.

The structure of lines along the slit (approximately along the major axis) is very interesting. Firstly, the maximum of emission for the high excitation lines such as [OIII]5007 coincide with those of $\mathrm{H} \alpha$ and density, but shifted by about 1 arcsec with respect to the shock ionised lines. Finally, the low excitation ratios [OI]6300/H $\alpha,[\mathrm{NII}] 6583 / \mathrm{H} \alpha$ and [SII]6725/H $\alpha$ are very well correlated with each other and clearly anticorrelated with the high excitation ratio [OIII] $5007 / \mathrm{H} \beta$. 